Analytical Methods

\title{
Detection of adulteration of extra-virgin olive oil by chemometric analysis of mid-infrared spectral data
}

\author{
Gozde Gurdeniz, Banu Ozen* \\ Izmir Institute of Technology, Department of Food Engineering, TR-35430 Urla-Izmir, Turkey
}

\section{A R T I C L E I N F O}

\section{Article history:}

Received 18 February 2008

Received in revised form 30 December 2008

Accepted 22 February 2009

\section{Keywords:}

Olive oil

Adulteration

Chemometrics

Mid-infrared spectroscopy

\begin{abstract}
A B S T R A C T
This study focuses on the detection and quantification of extra-virgin olive oil adulteration with different edible oils using mid-infrared (IR) spectroscopy with chemometrics. Mid-IR spectra were manipulated with wavelet compression previous to principal component analysis (PCA). Detection limit of adulteration was determined as $5 \%$ for corn-sunflower binary mixture, cottonseed and rapeseed oils. For quantification of adulteration, mid-IR spectral data were manipulated with orthogonal signal correction (OSC) and wavelet compression before partial least square (PLS) analysis. The results revealed that models predict the adulterants, corn-sunflower binary mixture, cottonseed and rapeseed oils, in olive oil with error limits of $1.04,1.4$ and 1.32, respectively. Furthermore, the data were analysed with a general PCA model and PLS discriminant analysis (PLS-DA) to observe the efficiency of the model to detect adulteration regardless of the type of adulterant oil. In this case, detection limit for adulteration is determined as $10 \%$.

(c) 2009 Elsevier Ltd. All rights reserved.
\end{abstract}

\section{Introduction}

Adulteration of food products involves the replacement of highcost ingredients with lower grade and cheaper substitutes (Tay, Singh, Krishnan, \& Gore, 2002). Due to its sensory quality and nutritional benefits extra-virgin olive oil is often adulterated with less expensive oils. Adulteration of olive oil is a serious problem for regulatory agencies, oil suppliers and could also threat health of consumers. Actually, blend edible oils can be prepared only for suitable products, but if the resulting blend deviates from the mixture proportions given on the label, or if the blend is traded as genuine, it means the oil is adulterated (Ulberth \& Buchgraber, 2000). The edible oils widely employed in virgin olive oil adulteration can be lower quality olive oil (refined or pomace olive oil) or other vegetable or seed oils such as corn, peanut, cottonseed, sunflower, soybean and poppy seed oils (Harwood \& Aparicio, 2000). Turkey is one of the major olive oil producers in the World and cottonseed, rapeseed, sunflower and corn oils with lower market price are commonly used to adulterate olive oil.

There exist numerous methodologies to detect and quantify vegetable or seed oils in olive oil. Techniques involving application of chromatographic methods are commonly applied (Andrikopoulos, Giannakis, \& Tzamtzis, 2001; Christopoulou, Lazaraki, Komaitis, \& Kaselimis, 2004; Dionisi, Prodolliet, \& Tagliaferri, 1995; Harwood \& Aparicio, 2000).

\footnotetext{
* Corresponding author. Tel.: +90 232750 6319; fax: +90 2327506196 .

E-mail addresses: banuozen@iyte.edu.tr, ozen_banu2@yahoo.com (B. Ozen).
}

However, methods of food adulteration have become more sophisticated due to its economic profits. There is an increasing demand for the development of new rapid and sensitive methods instead of traditional time-consuming and expensive analysis techniques. There are several studies about new emerging methods mainly focusing on this subject. One of these studies involves with the use of headspace autosampler directly coupled to a mass spectrometer (ChemSensor) to detect hazelnut oil adulteration (Peňa, Cárdenas, Gallego, \& Valcárcel, 2005). Total synchronous fluorescence (TSyF) spectroscopy was also employed to differentiate virgin olive oil from olive-pomace, corn, sunflower, soybean, rapeseed and walnut oils with PLS (Poulli, Mousdis, \& Georgiou, 2007). In another study, analysis of sunflower, corn, peanut and coconut oil adulterated olive oil by gas chromatography-mass spectrometry in combination with class analogy and $K$ nearest neighbours resulted in prediction ability higher than $91 \%$ for adulterant detection and $88 \%$ for type of adulterant identification ( $\mathrm{Ca}$ pote, Jiménez, \& Luque de Castro, 2007). Moreover, an electronic nose based on metal oxide semiconductor sensors was employed for the determination of olive oil adulteration and supplied 95\% precision (Oliveros et al., 2002).

Application of spectroscopy which includes IR and Raman techniques combined with chemometric methods is a relatively new approach to determine authenticity of olive oil. Employment of spectroscopic methods for the detection of authentication of vegetable oils has firstly emerged in the middle of 90s (Baeten, Meurens, Morales, \& Aparicio, 1996; Lai, Kemsley, \& Wilson, 1994, 1995; Wesley, Barnes, \& McGill, 1995). Many further studies were performed on the issue. Near-infrared (NIR), mid-infrared, 
and Raman spectroscopic techniques can quantify the amount of olive-pomace oil adulteration in extra-virgin olive oil $(0 \%$ and $100 \%$ in $5 \%$ increments by weight) with $R^{2}$ value higher than 0.99 (Yang \& Irudayaraj, 2001) for each technique. Christy, Kasemsumran, Du, and Ozaki (2004) studied NIR spectroscopy to detect and quantify adulteration of olive oil with soybean, sunflower, corn, walnut and hazelnut oils which resulted in error limits of $\pm 0.57 \%$ (corn oil), $\pm 1.32 \%$ (sunflower oil), $\pm 0.96 \%$ (soybean oil), $\pm 0.56 \%$ (walnut oil) and $\pm 0.57 \%$ (hazelnut oil). Fourier-transform infrared (FT-IR) spectroscopy equipped with a ZnSe-ATR accessory was able to detect adulteration of virgin olive oil with hazelnut oil at levels of $25 \%$ and higher (Ozen \& Mauer, 2002). Baeten et al. (2005) obtained better results in their study which involves application of Raman and MIR spectroscopies for determination of the level of hazelnut oil in olive oil (up to level of 8\%). Analysis of FTIR data with discriminant analysis allowed classification of olive oil and sunflower adulterated olive oil samples $(20 \mathrm{ml} / \mathrm{l})$ as pure and adulterated. In the same study, PLS model developed to determine the level of mixing resulted in $R^{2}$ value of validation set as 0.974 , which indicated the success of the model (Tay et al., 2002). Vlachos et al. (2006) also studied the determination of olive oil adulteration with vegetable oils using FT-IR and detection limit for olive oil adulteration is $9 \%$ if the adulterant is corn oil or sesame seed oil while it is lower (6\%) if the adulterant is sunflower or soybean oil.

Adulteration of olive oil with combination of several oils has become a problem for Turkish olive oil producers and consumers. Especially corn-sunflower mixture has been used to adulterate extra-virgin olive oil. There are a limited number of published studies focusing specifically on the issue of multiple blended oils used to adulterate EVOO. A chromatographic approach with PLS was applied for determination of cottonseed, olive, soybean and sunflower ternary and quaternary edible oil mixtures (Hajimahmoodi et al., 2005). The relative standard error for each oil in mixed samples was specified as less than $10 \%$. As the only example of spectroscopic approach, Özdemir and Öztürk (2007) focused on quantification of binary and tertiary adulteration of olive oil with sunflower and corn oil using NIR in conjunction with genetic inverse least square which resulted in SEP ranged between $1.42 \%$ and $6.38 \%$ ( $\mathrm{vol} / \mathrm{vol}$ ) for the ternary mixtures of olive, sunflower and corn oil. However, there is not any study involving the use of mid-IR spectroscopy for determination of ternary adulteration of olive oil.

Also, it is important to be able to detect olive oil adulteration regardless to the type of adulterant. Exceptionally, Capote et al. (2007) used gas chromatography-mass spectrometry data manipulated with soft independent modeling of class analogy (SIMCA) and $K$ nearest neighbours (KNN) to identify the oil used to adulterate olive oil and were able to reach $88 \%$ correct type of adulterant identification.

The aim of this study is to demonstrate the applicability of midIR spectroscopy as a rapid analysis method to detect and quantify adulteration of extra-virgin olive oil with vegetable oils (rapeseed, cottonseed and corn-sunflower binary mixture) by constructing (1) three independent models for cottonseed, rapeseed and sunflower-corn binary oil mixture adulterated olive oil samples and (2) overall model including all adulterated samples.

\section{Materials and methods}

\subsection{Samples}

Extra-virgin olive oil samples which belong to 25 different locations of Aegean region of Turkey were obtained from the same olive oil producer. The samples were kept in dark glass bottles and stored at $8^{\circ} \mathrm{C}$. Spectra of oils were obtained within two months after receiving the oils from producer.

While mainly Ayvalik variety is cultivated in North Aegean region, Memecik is the dominant variety in South part of Aegean region. Corn, sunflower, rapeseed and cottonseed oils were purchased from stores and blended with olive oil samples. For cottonseed and rapeseed oils adulteration, four olive oil samples belonging to north were blended with rapeseed and cottonseed oils at 2-20\% (vol/vol). For corn-sunflower oil adulteration, corn-sunflower oil binary mixtures were prepared at different concentrations (0-100\% vol/vol) and mixed with blends of four commercial olive oil samples produced in the North of Turkey at $2-20 \%$ ( vol $/ \mathrm{vol}$ ). Composition of each corn-sunflower adulterated olive oil sample was presented in Table 1.

\subsection{FT-IR analysis}

All infrared spectra $\left(4000-650 \mathrm{~cm}^{-1}\right)$ were acquired with a Perkin Elmer Spectrum 100 FT-IR spectrometer (Perkin Elmer Inc., Wellesley, MA). This instrument was equipped with a horizontal attenuated total reflectance (HATR) sampling accessory (ZnSe crystal) and a deuterated tri-glycine sulphate (DTGS) detector.

HATR accessory was used to collect the spectral data of oil. The resolution was set at $2 \mathrm{~cm}^{-1}$ and the number of scans collected for each spectrum was 128 . ZnSe crystal was cleaned with hexane in between sample runs. Measurements were conducted duplicate or triplicate for each olive oil sample.

\subsection{Multivariate analysis}

Data analysis was performed using multivariate statistical methods with SIMCA software (Umetrics, Sweden). 3620-2520 and $1875.5-675 \mathrm{~cm}^{-1}$ regions of FT-IR spectra were used in the analysis.

In order to transform the data into a form suitable for PCA and PLS, the data is often pre-treated. Within this concept spectral data was scaled and mean centered. Also, wavelet analysis as a widely used spectral compression technique preferentially employed before application of PCA and PLS (Eriksson et al., 2004; Trygg \& Wold, 1998). Its main abilities are compressing and de-noising complicated signals. Daubechies-10 was chosen amongst wavelet functions. PCA is a multivariate projection method designed to extract and display the systematic variation in a data matrix X and can also be used to develop a modeling technique called SIMCA which is one of the most commonly used class-modeling tools in chemometrics. In SIMCA, PCA is performed for each class

Table 1

Percentages of sunflower, corn and olive oil in 2-20\% adulterated mixtures.

\begin{tabular}{lll}
\hline Sunflower oil (\%) & Corn oil (\%) & Olive oil (\%) \\
\hline 1 & 1 & 98 \\
2 & 0 & 98 \\
0 & 2 & 98 \\
2.5 & 2.5 & 95 \\
5 & 0 & 95 \\
0 & 5 & 95 \\
5 & 5 & 90 \\
10 & 0 & 90 \\
0 & 10 & 90 \\
7.5 & 7.5 & 85 \\
10 & 5 & 85 \\
5 & 10 & 85 \\
10 & 10 & 80 \\
15 & 5 & 80 \\
5 & 15 & 80 \\
20 & 0 & 80 \\
0 & 20 & 80 \\
\hline
\end{tabular}


separately and this results in a PC model for each class (Massart, Vanbeginste, Deming, Michotte, \& Kaufman, 1988). A class space is built whose boundary discriminates between the samples fitting the class model and the samples that cannot be considered as belonging to the studied class. Mathematical model of the class should be defined and confidence interval that encloses the model should be determined (Lanteri, Armanino, Perri, \& Palopoli, 2002).

For quantification issues, whole observation data set was divided into calibration and validation sets. Determination of sunflower-corn, cottonseed and rapeseed oil amount (\%) in olive oil was performed by PLS analysis, which relates FT-IR absorbance of each adulterated sample (X block) with the percentages of adulterant oil (Y block). In quantification studies, OSC in combination with wavelet analysis was applied on spectral data. OSC is a signal correction technique and constructs a filter that removes the part definitely unrelated to Y from the spectral matrix X (Wold, Antti, Lindgren, \& Öhman, 1998). The ability of the PLS model was inspected with validation set. It is important to accurately determine the number of components that should be included in the model that is linked to the difference between the degree of fit and the predictive ability. Degree of fit increases as the number of components increases but predictive ability does not increase after a cer-

\section{(a)}

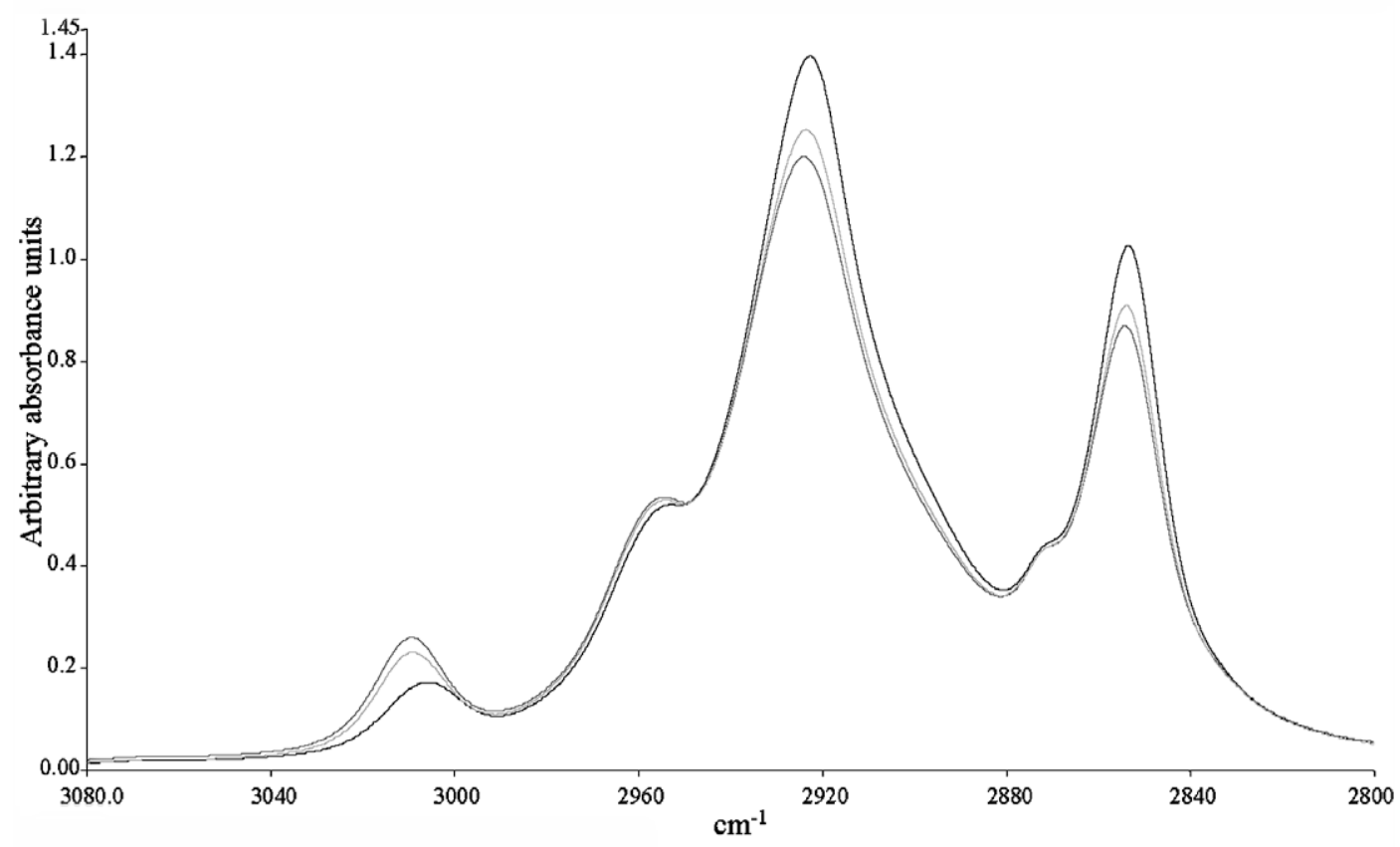

(b)

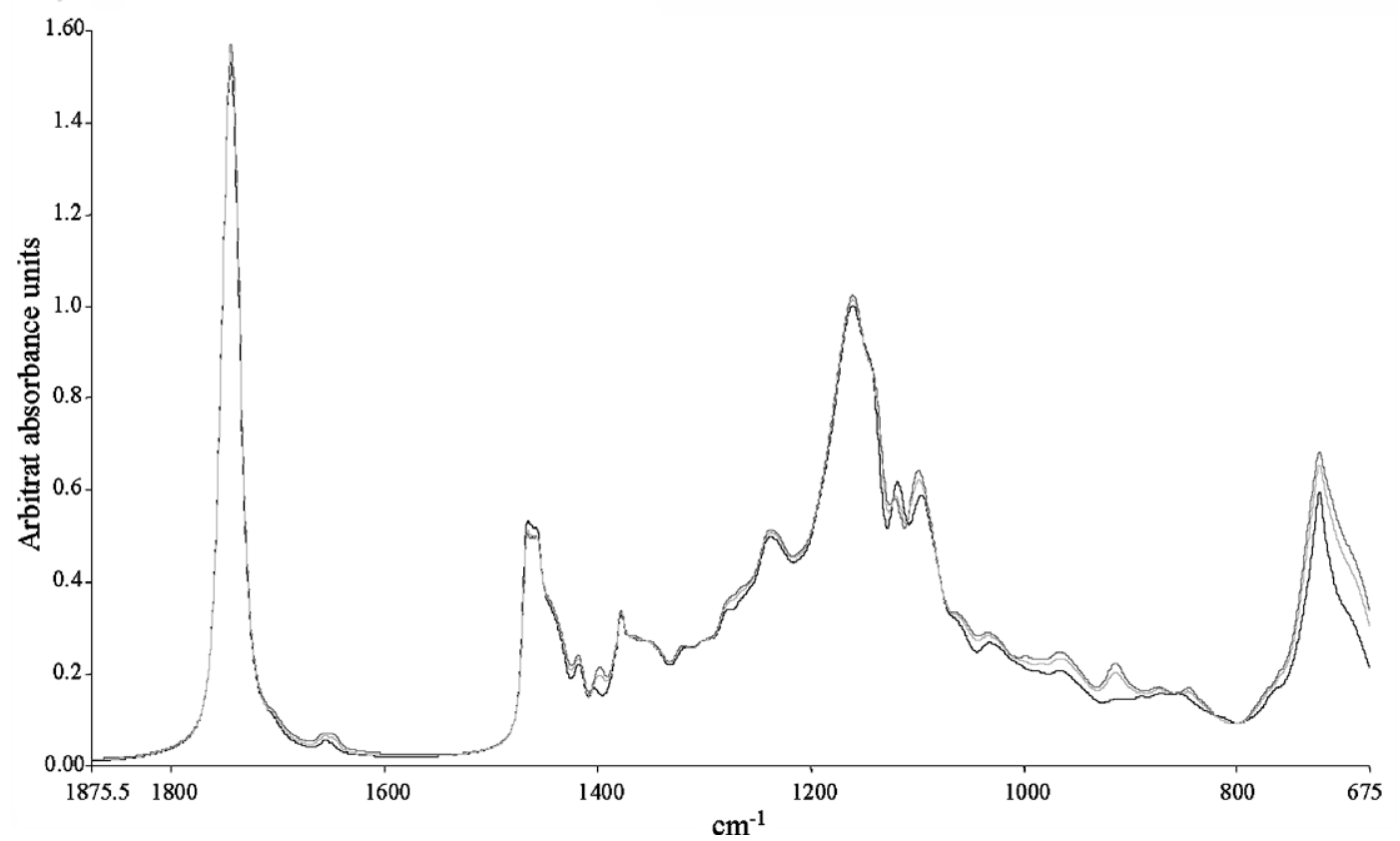

Fig. 1. The spectra of olive oil, corn oil and sunflower oil around (a) $3080-2800 \mathrm{~cm}^{-1}$ and (b) $2875.5-675 \mathrm{~cm}^{-1}$ region. ( - olive oil, - sunflower and corn oils, - cottonseed and rapeseed oils). 
tain model complexity. So, it is important to reach an optimal balance between fit and predictive ability. The predictability of the models was tested by computing the standard error of calibration (SEC) for the calibration data set and the standard error of prediction (SEP) for the validation data set:

$\mathrm{SEC}=\sqrt{\frac{\sum_{i=1}^{m}\left(\hat{Y}_{i}-Y_{i}\right)^{2}}{M-1}}$

$\mathrm{SEP}=\sqrt{\frac{\sum_{i=1}^{n}\left(\hat{Y}_{i}-Y_{i}\right)^{2}}{N}}$

Lower the error values for the selected data set, better the performance of the model. As expected, the model tends to have smaller $R$ and larger error values during prediction.

PLS-DA is a modified form of PLS applied in classification and discrimination problems. The main difference of PLS-DA from SIMCA is that a separate PCA model is constructed for each class using
SIMCA whereas PLS-DA makes one model covering many classes (Eriksson et al., 2004).

\section{Results and discussions}

From the 25 olive oil samples cultivated and produced in different locations of the Aegean region, four olive oil samples from the Northern regions were selected randomly, mixed and used as a base for the adulterated test mixtures. In general, mainly the Ayvalik olive variety is cultivated in the North Aegean region and Memecik is the dominant olive variety cultivated in the Southern part of this region and these varieties have major economic importance in Turkey.

Mid-IR spectroscopy as a rapid and non-destructive analysis tool was employed to detect adulteration. Olive oil spectra was compared with the spectra of other oil samples. Fig. 1 presents typical spectra of olive, sunflower, corn, cottonseed and rapeseed oil samples. Two distinct wavelength intervals with notable peaks were provided in Fig. 1 to visualize the spectral differences. Peaks around $2800-3080 \mathrm{~cm}^{-1}$ are due to hydrogen stretching mode. Large peak
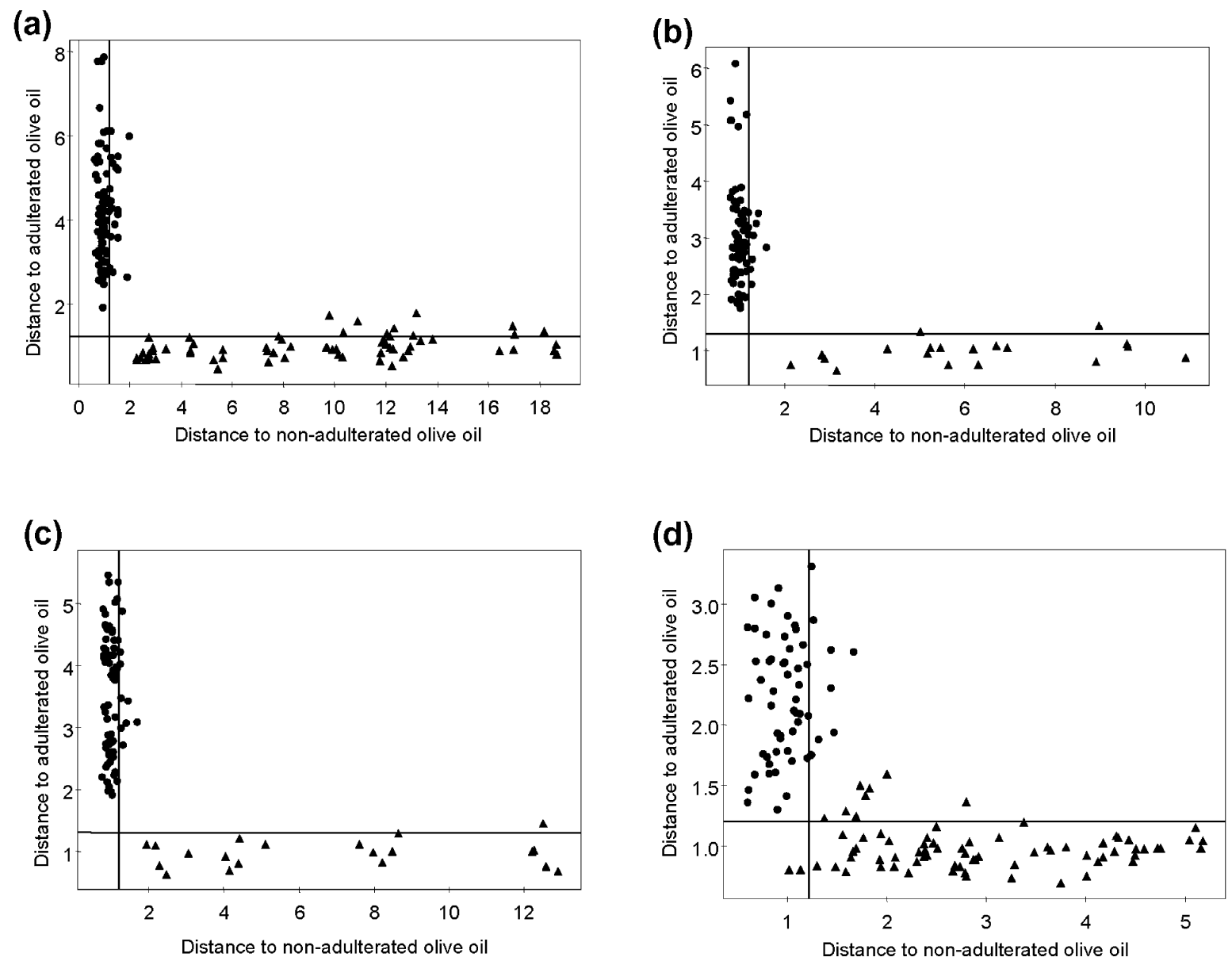

Fig. 2. Coomans' plots of (a) cottonseed oil (5-20\%), (b) rapeseed oil (5-20\%) and (c) corn-sunflower binary oil mixture (5-20\%) versus non-adulterated olive oil samples ( non-adulterated, $\boldsymbol{\Delta}$ adulterated olive oil samples).

Table 2

PLS statistics for calibration and validation sets.

\begin{tabular}{|c|c|c|c|c|c|c|c|}
\hline Adulterant & Number of PCs & Explained variation (\%) & Predictive ability (\%) & $R^{2}$ (calibration) & SEC & $R^{2}$ (validation) & SEP \\
\hline Corn-sunflower & 2 & 99 & 98.9 & 0.99 & 0.78 & 0.98 & 1.04 \\
\hline Cottonseed & 3 & 99.2 & 97.9 & 0.99 & 0.49 & 0.95 & 1.4 \\
\hline Rapeseed & 2 & 99.4 & 99.1 & 0.994 & 0.48 & 0.93 & 1.32 \\
\hline
\end{tabular}


between 1700 and $1800 \mathrm{~cm}^{-1}$ could be attributed to $\mathrm{C}=0$ stretching, and $\mathrm{C}-\mathrm{O}-\mathrm{C}$ stretching and $\mathrm{C}-\mathrm{H}$ bending in the region of $900-$ $1400 \mathrm{~cm}^{-1}$ could be easily observed (Tay et al., 2002). Changes in the intensities of spectral bands of different oils are related to the composition of molecular bonds absorbing at those regions. There exists a notable difference between olive oil and adulterant oils in the peak between 3010 and $3000 \mathrm{~cm}^{-1}$ resulting from the $\mathrm{C}-\mathrm{H}$ stretching vibration of the cis-double bonds $(=\mathrm{CH})$. The intensity of this band changes slightly for each adulterant oil and olive oil significantly differs from them. The height of large peak around $2923 \mathrm{~cm}^{-1}$ is attributed to symmetrical stretching vibration of the aliphatic $\mathrm{CH}_{2}$ group which varies between oils with higher absorbance values for olive oil. The availability of peak heights around $3006 \mathrm{~cm}^{-1}$ with the contribution of band around $2925 \mathrm{~cm}^{-1}$ to detect olive oil adulteration was illustrated by Vlachos et al. (2006). The band around $2853 \mathrm{~cm}^{-1}$ due to symmetrical stretching vibration of the aliphatic $\mathrm{CH}_{2}$ group changes with respect to type of oil. There are also differences between the intensities of bands in Fig. 1b. A remarkable variation is observed in the peak heights around $1377 \mathrm{~cm}^{-1}$ resulting from bending vibrations of $\mathrm{CH}_{2}$ groups. Adulterant oils exhibit some intensity in the band around 913$914 \mathrm{~cm}^{-1}$ whereas intensity and position of band is different for olive oil showing very low or no intensity. Guillén and Cabo (1999) employed this band to detect and quantify adulteration. Spectral regions which do not contain any relevant information were excluded in the further analysis and bands between $3120-2520 \mathrm{~cm}^{-1}$ and $1875.5-675 \mathrm{~cm}^{-1}$ were selected to be employed.

(a)

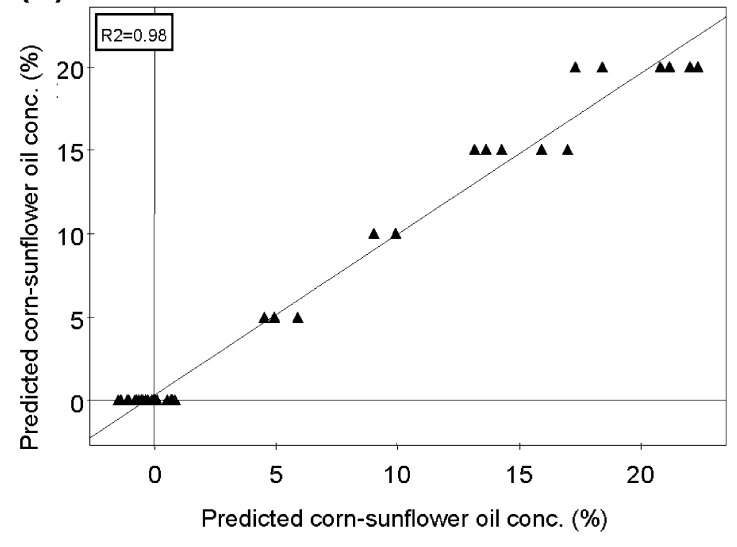

(c)

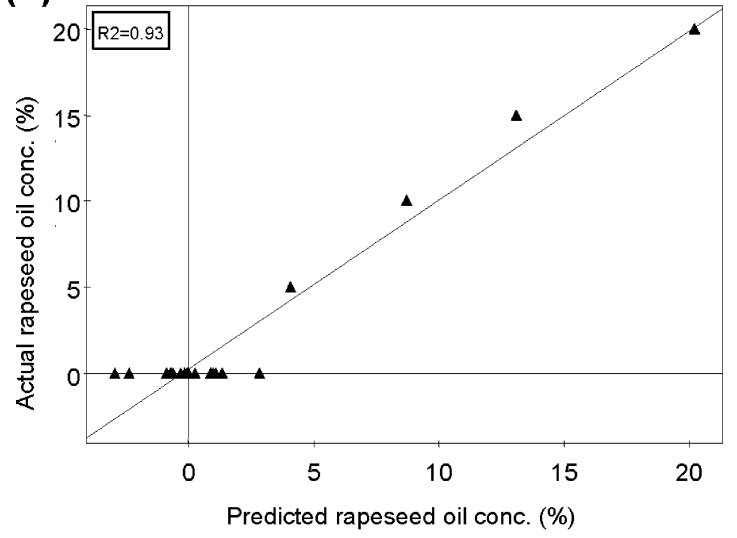

Adulterated samples were grouped according to type of oil used in the adulteration. PCA was performed separately for each adulterated group with pure olive oil sample. Pure olive oil samples include 25 olive oil samples from North and South Aegean regions. Olive oil samples, selected from different varieties and locations, bring variability to the pure olive oil class. Actually, it is important to be able to decide whether adulterated samples can be differentiated from pure olive oil samples by overcoming the variability existing between the samples and pure olive oil class. Then, we would be able to test the efficiency of the model in differentiation of adulterated samples from olive oil belonging to different varieties and geographical regions. To increase the computational efficiency and also to enhance the classification studies wavelet analysis was applied to spectral data as a compression technique. Scores plot (not shown) revealed that $2 \%$ adulterated samples of cottonseed, rapeseed oils and corn-sunflower binary oil mixture are plotted too close to pure olive oil samples which hinder discrimination. Thus, they are excluded in the further analysis. Then, Coomans' plot is constructed to more clearly visualize the discrimination of adulterated samples from pure olive oil samples. Fig. 2 displays Coomans' plots of cottonseed (5-20\%), rapeseed (5-20\%) oils and corn-sunflower binary oil mixture (5-20\%), versus pure olive oil samples. Coomans' plot of cottonseed and rapeseed oil adulteration exhibited quite successful discrimination of olive oil samples from adulterated samples (Fig. 2a and b). Besides, each grouping of sample points in non-adulterated class model represents different adulteration percentages in ascending order from
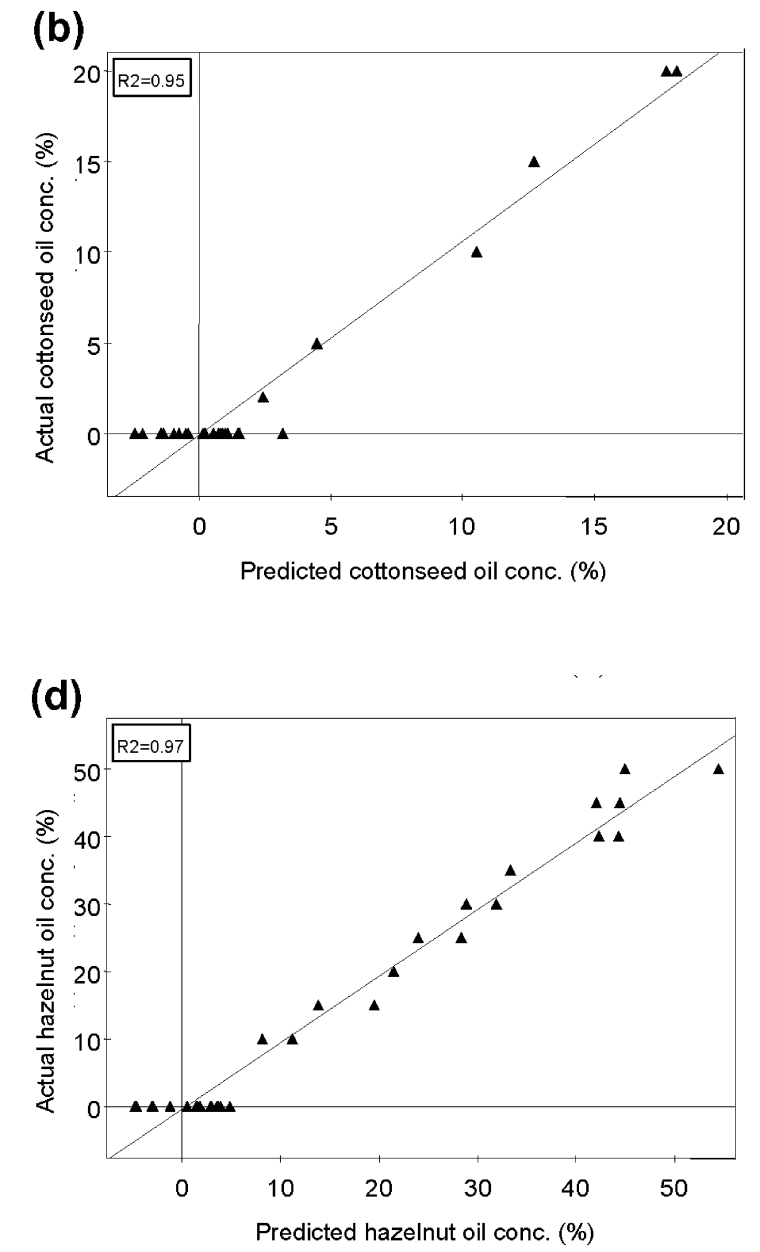

Fig. 3. Actual versus predicted percentages of (a) cottonseed oil, (b) rapeseed oil and (c) corn-sunflower binary oil mixture in olive oil for calibration and validation sets ( calibration set and $\bigcirc$ : validation set). 
left to right for rapeseed adulteration. Also, Coomans' plot of cornsunflower adulteration reveals discrimination of adulterated samples from non-adulterated oil samples. In a previous study, analysis of different varieties of olive oils in Turkey with mid-IR spectra and PCA resulted in tolerable discrimination between Ayvalik and Memecik olive varieties (Gurdeniz, Ozen, \& Tokatli, 2008). Consequently, the model constructed here appears to be more efficient in that it can group significantly different olive oil varieties together and discriminate adulterated olive oil samples.

There are other studies involving the use of sunflower-olive oil and corn-olive oil binary blends to detect olive oil adulteration with FT-IR. Tay et al. (2002) were able to discriminate olive oil samples from sunflower adulterated samples at levels between $2 \%$ and $10 \%$. Also, results of FT-IR analysis indicated that the detection limit for olive oil adulteration was $9 \%$ if the adulterant is only corn oil while it was lower (6\%) if the adulterant is sunflower oil (Vlachos et al., 2006). However, each of these studies includes detection of adulteration from binary mixtures. As far as the authors are aware, no published work has focused on the detection of ternary olive oil adulteration except the study of Özdemir and Öztürk (2007) whose study focused on quantification of binary and ternary adulteration of olive oil with sunflower and corn oil using NIR in conjunction with genetic inverse least square. Overall, SEP ranged between $2.49 \%$ and $2.88 \%$ ( $\mathrm{vol} / \mathrm{vol}$ ) for the binary mixtures of olive and sunflower oil whereas it was between 1.42 and $6.38 \%$ (vol/vol) for the ternary mixtures of live, sunflower and corn oil.

Quantification of adulterant oil content in adulterated oil samples was performed using PLS algorithm. The same data set except $2 \%$ adulterant level was used in PLS analysis. The samples of all the adulterated and pure olive oils were randomly divided into a calibration and a validation set. OSC was applied to remove systematic variation in $\mathrm{X}$ (spectral data) that is not related with $\mathrm{Y}$ (per cent adulteration). In addition, wavelength compression was selected to increase the efficiency and speed. Fig. 3 displays actual versus predicted percentages of cottonseed, rapeseed oils and corn-sunflower binary oil mixture in olive oil for calibration and validation sets. Difference between the actual and predicted adulterant oil concentration is small indicating the success of PLS model. The statistics of PLS of calibration and validation sets are given in Table 2 . High $R^{2}$ value and low SEP and SEC values demonstrate high predictive ability of each model. Christy et al. (2004) employed NIR spectra with PLS to detect olive oil adulteration with corn, sunflower and hazelnut oils and error terms were determined as 1.32 and, 0.57 for sunflower and corn oils at levels of $0-100 \%$.

In the last part of the study, adulterated oils regardless of the type of adulterant were grouped as one adulterant class and subjected to PCA to establish if authentic olive oil samples could be distinguished from adulterated samples. Observations were classified as adulterated including spectra of rapeseed and cottonseed oils and corn-sunflower binary oil mixture and pure olive oil samples. It is important to determine if this model would be able discriminate an olive oil sample adulterated with an unknown adulterant from pure olive oils. To construct Coomans' plot (Fig. 4a) PCA was performed on adulterated and pure classes separately. Modeling adulterated oil samples together means more variable chemical information making them more difficult to be placed in the same group. Thus, $2 \%$ and $5 \%$ adulterated olive oil samples are placed so close to pure olive oil samples according to scores plot (not shown). Therefore, the samples (2\% and $5 \%$ adulterated) were excluded from the data set. According to Coomans' plot, most samples of adulterated and pure samples are correctly placed in their region. However, few samples are plotted in the region where they can not be classified as pure or adulterated. Same classified data was also manipulated with PLS-DA and scores plot is shown in Fig. 4b. Pure and adulterated samples are placed in right and left hemispheres, (Fig. 4b) indicating quite successful discrim-
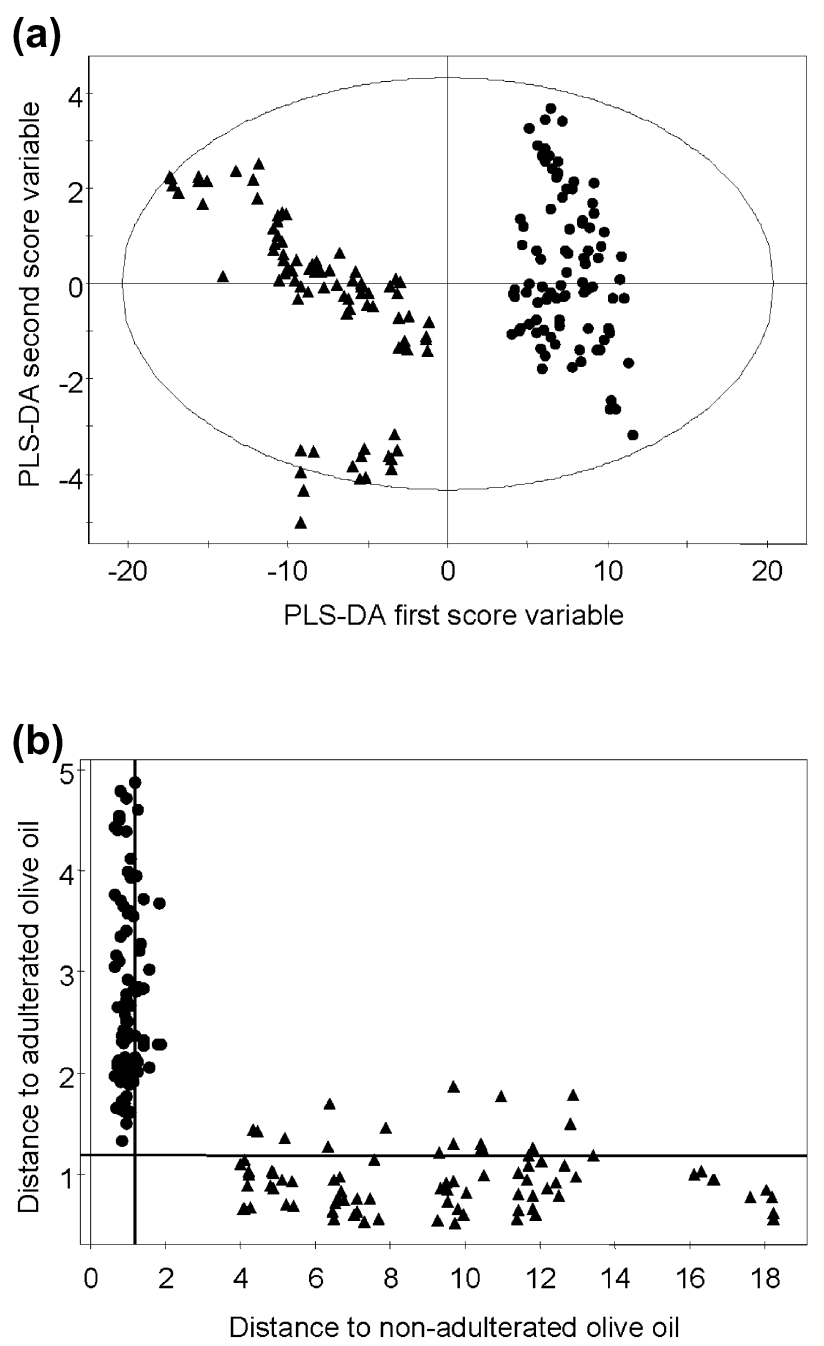

Fig. 4. (a) Coomans' plot and (b) scores plot of PLS-DA for corn-sunflower binary mixture, cottonseed and rapeseed adulterated versus pure olive oil samples ( nonadulterated, $\boldsymbol{\Delta}$ adulterated olive oil samples).

ination using PLS-DA. SIMCA and PLS-DA are both supervised pattern recognition techniques that aim to establish classification. However, PLS-DA classifies samples into one of the given categories, even if they do not belong to any of them. On the other hand, SIMCA visualized with Coomans' plot is able to discriminate nonmembers (Berrueta, Alonso-Salces, \& Héberger, 2007). Therefore, visual discrimination seems to be better for PLS-DA than Coomans' plot. However, samples that do not belong to any group can also be displayed by Coomans' plot (Fig. 4a). There are studies involving the application of other analytical and chemometric methods to discriminate olive oil from set of different adulterant oils. Availability of chromatographic profiles with SIMCA model to distinguish between pure olive oil samples and those adulterated with one of the vegetable oils (sunflower, corn, peanut and coconut oils) was illustrated (Capote et al., 2007). Also, NIR spectra manipulated with PCA was able to classify adulterated olive oil samples with respect to type of adulterant oil (Christy et al., 2004). Amongst these and similar studies, mid-IR spectroscopy data was not employed before to construct one model including adulterated olive oil samples using different oils. In fact one can not know the type of adulterant used to adulterate an olive oil sample and employment of such an overall model can be reasonable.

In conclusion, manipulation of mid-IR spectra with chemometrics leads to detection and quantification of olive oil adulteration. 
Besides, detecting potential adulterants, cottonseed and rapeseed oils, in olive oil at a level of $5 \%$, detection limit of binary mixture of corn-sunflower oil in olive oil is also found out as 5\%. Furthermore, mid-IR spectroscopy with chemometrics has the potential to detect adulteration of olive oil regardless of the type of adulterant oil but at a higher adulteration detection level. Quantification studies resulted in calibration curves with $R^{2}$ values around 0.9 .

In the further studies, this work can be expanded by including samples from other regions and other parts of the World and these olive oil samples can be mixed with common adulterant oils. It is likely that the use of Mid-IR results from an expanded vegetable oil reference data set will result in more comprehensive models to improve the detection and quantification of adulteration of olive oils.

\section{Acknowledgements}

Authors would like to thank Union of Taris Olive and Olive Oil Co-operatives for providing olive oil samples and Dr. Figen Tokatli for assistance in statistical analysis. This study was performed as part of CODA (MIRG-CT-2005-029134) project supported by EU Marie Curie Reintegration Grant.

\section{References}

Andrikopoulos, N. K., Giannakis, I. G., \& Tzamtzis, V. (2001). Analysis of olive oil and seed oil triglycerides by capillary gas chromatography as a tool for the detection of the adulteration of olive oil. Journal of Chromatographic Science, 39, 137-145.

Baeten, V., Meurens, M., Morales, M. T., \& Aparicio, R. (1996). Detection of virgin olive oil adulteration by Fourier transform Raman spectroscopy. Journal of Agricultural Food Chemistry, 44(8), 2225-2230.

Baeten, V., Pierna, J. A. F., Dardenne, P., Meurens, M., García-González, D. L., \& Aparicio-Ruiz, R. (2005). Detection of the presence of hazelnut oil in olive oil by FT-Raman and FT-MIR spectroscopy. Journal of Agricultural and Food Chemistry, 53, 6201-6206.

Berrueta, L. A., Alonso-Salces, R. M., \& Héberger, K. (2007). Supervised pattern recognition in food analysis. Journal of Chromatography A, 1158, 196-214.

Capote, F. P., Jiménez, J. R., \& Luque de Castro, M. D. (2007). Sequential (step-bystep) detection, identification and quantitation of extra virgin olive oil adulteration by chemometric treatment of chromatographic profiles. Analytical and Bioanalytical Chemistry, 388, 1859-1865.

Christopoulou, E., Lazaraki, M., Komaitis, M., \& Kaselimis, K. (2004). Effectiveness of determinations of fatty acids and triglycerides for the detection of adulteration of olive oils with vegetable oils. Food Chemistry, 84, 463-474.

Christy, A. A., Kasemsumran, S., Du, Y., \& Ozaki, Y. (2004). The detection and quantification of adulteration in olive oil by near-infrared spectroscopy and chemometrics. Analytical Sciences, 20, 935-940.

Dionisi, F., Prodolliet, J., \& Tagliaferri, E. (1995). Assessment of olive oil adulteration by reversed-phase high-performance liquid chromatography/amperometric detection of tocopherols and tocotrienols. Journal of the American Oil Chemists Society, 72, 1505-1511.

Eriksson, L., Antti, H., Gottfries, J., Holmes, E., Johansson, E., Lindgren, F., et al. (2004). Using chemometrics for navigating in the large data sets of genomics, proteomics, and metabonomics (gpm). Analytical and Bioanalytical Chemistry, $380,419-429$.

Guillén, M. D., \& Cabo, N. (1999). Usefulness of the frequencies of some Fourier transform infrared spectroscopic bands for evaluating the composition of edible oil mixtures. Fett/Lipid, 101, 71-76.

Gurdeniz, G., Ozen, B., \& Tokatli, F. (2008). Classification of Turkish olive oils with respect to cultivar, geographic origin and harvest year, using fatty acid profile and mid-IR spectroscopy. European Food Research and Technology, 227, $1275-1281$

Hajimahmoodi, M., Vander Heyden, Y., Sadeghi, N., Jannat, B., Oveisi, M. R., \& Shahbazian, S. (2005). Gas-chromatographic fatty-acid fingerprints and partial least squares modeling as a basis for the simultaneous determination of edible oil mixtures. Talanta, 66, 1108-1116.

Harwood, J., \& Aparicio, R. (2000). Handbook of olive oil. Analysis and properties. Gaithersburg: Aspen.

Lai, Y. W., Kemsley, E. K., \& Wilson, R. H. (1994). Potential of Fourier transform infrared spectroscopy for the authentication of vegetable oils. Journal of Agricultural Food Chemistry, 42, 1154-1159.

Lai, Y. W., Kemsley, E. K., \& Wilson, R. H. (1995). Quantitative analysis of potential adulterants of extra virgin olive oil using infrared spectroscopy. Food Chemistry, 53, 95-98.

Lanteri, S., Armanino, C., Perri, E., \& Palopoli, A. (2002). Study of oils from Calabrian olive cultivars by chemometric methods. Food Chemistry, 76, 501-507.

Massart, D. L., Vanbeginste, B. G. M., Deming, S. N., Michotte, Y., \& Kaufman, L. (1988). Chemometrics: A textbook. Amsterdam: Elsevier Science.

Oliveros, M. C. C., Pavón, J. L. P., Pinto, C. G., Laespada, M. E. F., Cordero, B. M., \& Forina, M. (2002). Electronic nose based on metal oxide semiconductor sensors as a fast alternative for the detection of adulteration of virgin olive oils. Analytica Chimica Acta, 459, 219-228.

Özdemir, D., \& Öztürk, B. (2007). Near infrared spectroscopic determination of olive oil adulteration with sunflower and corn oil. Journal of Food and Drug Analysis, 15(1), 40-47.

Ozen, B. F., \& Mauer, L. J. (2002). Detection of hazelnut oil adulteration using FT-IR spectroscopy. Journal of Agricultural Food Chemistry, 50, 3898-3901.

Peňa, F., Cárdenas, S., Gallego, M., \& Valcárcel, M. (2005). Direct olive oil authentication: Detection of adulteration of olive oil with hazelnut oil by direct coupling of headspace and mass spectrometry, and multivariate regression techniques. Journal of Chromatography Analysis, 1074, 215-221.

Poulli, K. I., Mousdis, G. A., \& Georgiou, C. A. (2007). Rapid synchronous fluorescence method for virgin olive oil adulteration assessment. Food Chemistry, 150, 369-375.

Tay, A., Singh, R. K., Krishnan, S. S., \& Gore, J. P. (2002). Authentication of olive oil adulterated with vegetable oils using Fourier transform infrared spectroscopy. LWT-Food Science and Technology, 35, 99-103.

Trygg, J., \& Wold, S. (1998). PLS regression on wavelet compressed NIR spectra Chemometrics and Intelligent Laboratory Systems, 42, 209-220.

Ulberth, F., \& Buchgraber, M. (2000). Authenticity of fats and oils. European Journal of Lipid Science Technology, 102, 687-694.

Vlachos, N., Skopelitis, Y., Psaroudaki, M., Konstantinidou, V., Chatzilazarou, A., \& Tegou, E. (2006). Application of Fourier transform-infrared spectroscopy to edible oils. Analytica Chimica Acta, 573-574, 459-465.

Wesley, I. J., Barnes, R. J., \& McGill, A. E. J. (1995). Measurement of adulteration of olive oils by near-infrared spectroscopy. Journal of the American Oil Chemists' Society, 72, 289-292.

Wold, S., Antti, H., Lindgren, F., \& Öhman, J. (1998). Orthogonal signal correction of near-infrared spectra. Chemometrics and Intelligent Laboratory Systems, 44, 175-185.

Yang, H., \& Irudayaraj, J. (2001). Comparison of near-infrared, Fourier transforminfrared, and Fourier transform-Raman methods for determining olive pomace oil adulteration in extra virgin olive oil. Journal of the American Oil Chemists' Society, 78, 889-895. 